\title{
Macroscopic Finite Element for a Single Lap Joint
}

\author{
Scott E. Stapleton ${ }^{*}$ and Anthony M. Waas. ${ }^{\dagger}$ \\ University of Michigan, Ann Arbor, MI, 48109, USA
}

\begin{abstract}
Macroscopic finite elements are elements with an embedded analytical solution used to carry out efficient, mesh independent finite element analysis. In the present study, this method of macro elements was applied to a single lap joint. The adherends were modeled as Euler-Bernoulli beams, and the adhesive layer assumed to be in a state of plane stress. The field equations were derived using the principle of minimum potential energy, and the resulting solutions for the displacement fields were used to generate shape functions and a stiffness matrix for a single finite element. Simplifying assumptions incorporated in the model development were evaluated by comparing with several corresponding 2-D finite element models with different joint parameters. The results showed that the derived macroelement results in considerable cost savings in computational modeling of structural systems that contain multiple lap joints.
\end{abstract}

\section{Introduction}

$\mathrm{I}$ $\mathrm{N}$ the aerospace industry, fiber reinforced polymer matrix laminated composites (FRPCs) are gaining increasing use and attention because of their high strength to weight ratios, among other factors. FRPCs perform much better with adhesive bonding rather than bolting or riveting because of their quasi-brittle nature ${ }^{1}$ and the ability of the bond to spread the load over a larger area leading to a lessening of stress concentration ${ }^{2}$. Therefore, accurate analysis of adhesively bonded joints is becoming more critical than ever

Adhesive joints have traditionally been analyzed using two methods: analytical models and finite element analysis ${ }^{3}$. Analytical methods have been utilized to extract efficient closed-form solutions to adhesive single lap joint stresses. Classical formulas have been introduced by Volkerson ${ }^{4}$, Goland and Reissner ${ }^{5}$ and Hart-Smith ${ }^{6}$. More recently, refined analytical studies, carried out by Mortensen and Thomsen ${ }^{7}$ and Delale, Erdogan, and Aydinoglu, ${ }^{8}$ have proven to be quite accurate in predicting stresses within adhesive joints. However, analytical methods are often limited by geometric assumptions used to obtain a closed form solution and are not as useful to designers for compiling vehicle-scale models that may contain multiple joints. Finite element analyses are widely utilized in industry, and can be used to assess joints with a wide variety of geometries and loading conditions. However, these methods can suffer from mesh dependence and a lack of efficiency, which is especially crippling for

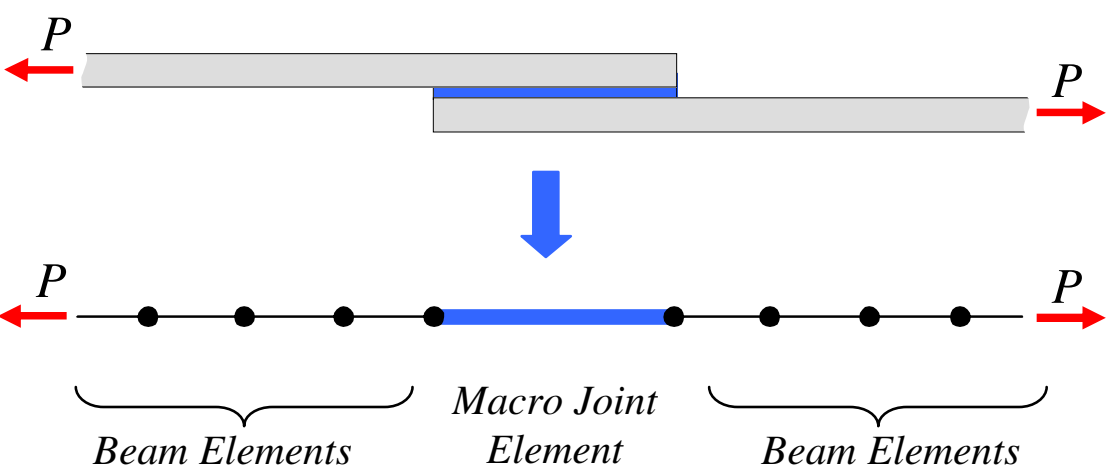

Figure 1. The present study replaces a complex single lap joint with one macro joint element. initial sizing analysis and full vehicle-scale models ${ }^{9}$. Therefore, a need exists to develop predictive tools for bonded joints that can seamlessly be coupled with large scale structural analyses without adding computational complexity. Such tools can be used to make quick meshindependent assessments of bonded composite joints. Currently, such a capability is lacking, and joint

\footnotetext{
${ }^{*}$ Graduate Student Research Assistant, Department of Aerospace Engineering. AIAA member.

${ }^{\dagger}$ Felix Pawlowski Collegiate Professor of Aerospace Engineering, Department of Aerospace Engineering. Associate Fellow, AIAA. Email: dcw@umich.edu
} 
assessment is typically performed late in the design cycle when structural changes that can lower the weight are much more difficult and expensive.

Gustafson and Waas ${ }^{3}$ have merged analytical and finite element methods in order to make efficient, mesh independent finite element analysis of double lap joints to use for initial design and macroscopic vehicle modeling. Analytical models were embedded into a single finite element with minimal analyst input. The current study extends this method to single lap joints (Fig. 1), which are more complex due to the eccentricity of the axial load path. Initially, the adherends are treated as Euler-Bernoulli beams, and the adhesive is modeled as a discrete bed of normal and shear springs. Extensions to include a nonlinear constitutive model for the adhesive in conjunction with inputs to cohesive zone finite element modeling ${ }^{10,11}$ is currently underway. The principle of minimum potential energy is used to obtain a closed-form solution of the adherend displacements, and these solutions are used to obtain shape functions and a local stiffness matrix for the macroscopic joint element. The entire joint can then be replaced by a single macro joint finite element, while the remaining structure (outside the joint) is modeled using standard structural elements, for instance beam elements (Fig. 1).

In this paper, simplifying assumptions incorporated in the development of a macro single lap joint element are evaluated by comparing the adhesive stress state against a 2-D FEM solution over a broad range of joint parameters to determine the geometric bounds of the simplifying assumptions. This trade study not only showed the effect of simplifying assumptions, but more importantly served as a validation study to show that the macroscopic element is accurate enough for modeling a wide range of joints in vehicle-scale models. Accuracy of the macroscopic joint element was found to be excellent, especially for thin, long adhesive layers, which are routinely used in aerospace applications of bonded FRPC joints.

\section{Analytical Formulation}

In order to create a macro element for a single lap joint, an analytical model of the joint was first implemented. The adhesive and adherends were assumed to be of an isotropic, linearly elastic material. Figure 2 illustrates the

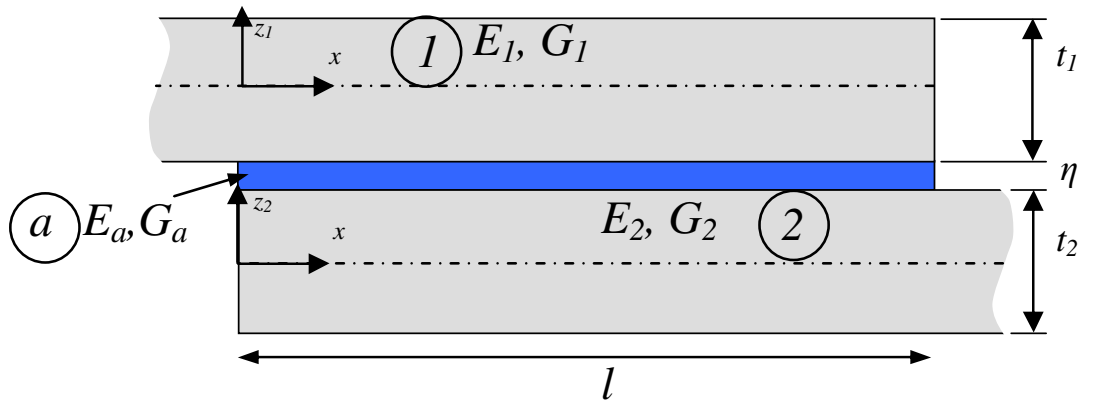

Figure 2. Geometric and material parameters for overlap region of a single lap joint.

$$
U=\frac{\int \sigma_{1 x x} \varepsilon_{1 x x} d V_{1}}{2}+\frac{\int \sigma_{2 x x} \varepsilon_{2 x x} d V_{2}}{2}+\frac{\int \sigma_{a x x} \varepsilon_{a x x} d V_{a}}{2}+\frac{\int \sigma_{a z z} \varepsilon_{a z z} d V_{a}}{2}+\frac{\int \tau_{a x z} \gamma_{a x z} d V_{a}}{2}
$$

where $\sigma_{\mathrm{ixx}}$ and $\varepsilon_{\mathrm{ixx}}$ represent the normal stress and strain in material $i$ ( 1 or 2 for the adherends, a for the adhesive) in the $x$ - direction, $\sigma_{\mathrm{azz}}$ and $\varepsilon_{\mathrm{azz}}$ are the normal stresses/strains in the adhesive in the $\mathrm{z}$ direction, $\tau_{\mathrm{axz}}$ and $\gamma_{\mathrm{axz}}$ represent the shear stress/strain in the adhesive on the $x z$ plane, and all integrals are taken over the volume, $V_{i}$ of material $i$.

It should be noted that many adhesive joints which bond FRPCs are made up of thin adherends which are relatively long in the $y$-direction, causing them to behave more like plates in cylindrical bending rather than beams. To model the adherends as wide plates in cylindrical bending, it is only a simple matter of replacing the modulus of elasticity, $E_{1}$ and $E_{2}$ with $E_{i} /\left(1-v_{i}^{2}\right), i=1,2$ and modeling the adhesive using plane strain, rather than plane stress assumptions.

The derivation of the strain energy is the same for adherends 1 and 2. Using Euler-Bernoulli beam theory, the displacements in the $x$-direction, $u_{l}\left(x, z_{1}\right)$ can be written as:

$$
u_{1}(x, z)=u_{1 \text { centerline }}(x)-z_{1}\left(\frac{d}{d x} w_{1 \text { centerline }}(x)\right)
$$


where $u_{\text {lcenterline }}(x)$ is the centerline displacement of adherend 1 in the $x$-direction, and $w_{\text {lcenterline }}(x)$ is the centerline displacement of adherend 1 in the $z$-direction.

For simplicity, $u_{l}$ will now be used to represent the axial displacement at the centerline of adherend 1 , and $w_{l}$ will represent the transverse displacement at the adherend centerline. In terms of centerline displacements, the internal strain energy in adherend $1, U_{l}$, becomes:

$$
U_{1}=\frac{1}{2} \int_{0}^{l} E A_{1}\left(\frac{d}{d x} u_{1}(x)\right)^{2}+E I_{1}\left(\frac{d^{2}}{d x^{2}} w_{1}(x)\right)^{2} d x
$$

where

$$
E A_{1}=E_{1} b t_{1} \text { and } E I_{1}=\frac{E_{1} b t_{1}^{3}}{12}
$$

The strain energy for the second adherend can be obtained using the same method.

A diagram of the notation scheme for the adhesive is shown in Fig. 3. It is assumed that the displacement varies linearly in the $z_{a}$-direction and that the adhesive and adherend are perfectly bonded at the interface. The displacements at the interface will be denoted by the subscript $i$. Assuming linear strain in the $z$-direction, the displacements of the adhesive can be written as

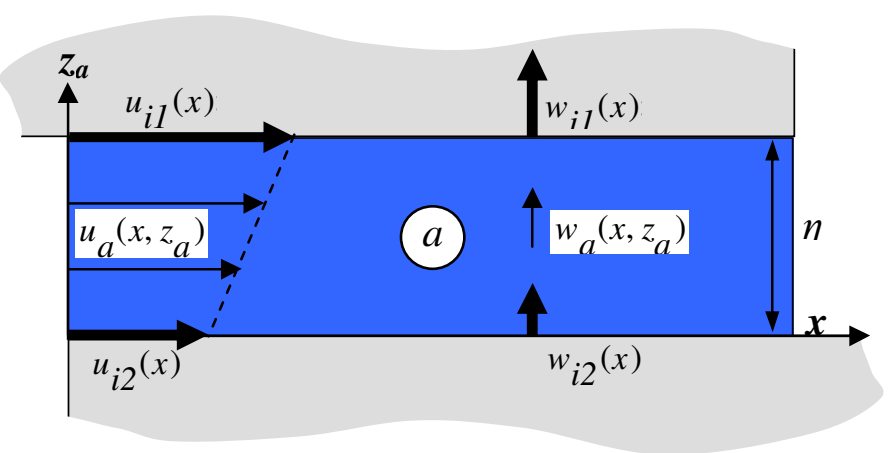

Figure 3. Schematic of the deformations in the adhesive.

$$
\begin{gathered}
u_{a}=u_{i 2}+\frac{z_{a}\left(u_{i 1}-u_{i 2}\right)}{\eta} \\
w_{a}=w_{i 2}+\frac{z_{a}\left(w_{i 1}-w_{i 2}\right)}{\eta} .
\end{gathered}
$$

For small strains, the strains in the adhesive can be written in terms of the adhesive displacements:

$$
\begin{gathered}
\gamma_{a x z}=\left(\frac{\partial}{\partial z_{a}} u_{a}\left(x, z_{a}\right)\right)+\left(\frac{\partial}{\partial x} w_{a}\left(x, z_{a}\right)\right), \\
\varepsilon_{a z z}=\frac{\partial}{\partial z_{a}} w_{a}\left(x, z_{a}\right), \text { and } \\
\varepsilon_{a x x}=\frac{\partial}{\partial x} u_{a}\left(x, z_{a}\right) .
\end{gathered}
$$

Assuming that the adhesive is in a state of plane stress, linear elasticity dictates that the stresses are:

$$
\tau_{a x z}=G_{a} \gamma_{a x z}
$$




$$
\begin{gathered}
\sigma_{a z z}=\frac{E_{a}\left(\varepsilon_{a z z}+v_{a} \varepsilon_{a x x}\right)}{1-v_{a}^{2}}, \text { and } \\
\sigma_{a x x}=\frac{E_{a}\left(\varepsilon_{a x x}+v_{a} \varepsilon_{a z z}\right)}{1-v_{a}^{2}}
\end{gathered}
$$

Now, the interface displacements are defined in terms of adherend centerline displacements using Euler-Bernoulli beam theory:

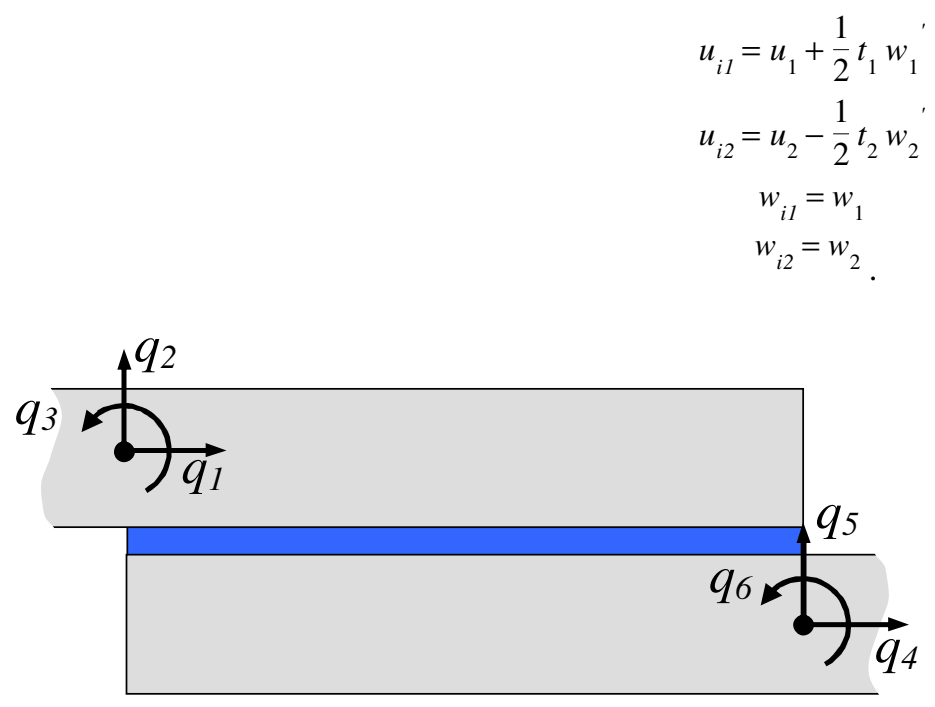

Figure 4. Prescribed nodal displacements used to obtain shape functions for the single lap joint.
Note that for simplicity, derivatives with respect to $x$ are denoted by the prime symbol and functions of $x$ are not indicated $(f(x) \rightarrow f)$ for the remainder of the paper. Using the principle of stationarity of potential energy, four fully coupled governing equilibrium differential equations were obtained from the energy expression. The governing equations were solved for the displacements $u_{l}, u_{2}, w_{l}$, and $w_{2}$. The prescribed nodal displacements, $q_{1}-q_{6}$ shown in Fig. 4, were applied as boundary conditions, thus resulting in equations describing the adherend centerline displacements in terms of the discrete nodal displacements. This resulted in shape functions, $N_{i, j}$, of the form:

$$
\left[\begin{array}{c}
u_{1} \\
w_{1} \\
u_{2} \\
w_{2}
\end{array}\right]=\left[\begin{array}{llllll}
N_{1,1} & N_{1,2} & N_{1,3} & N_{1,4} & N_{1,5} & N_{1,6} \\
N_{2,1} & N_{2,2} & N_{2,3} & N_{2,4} & N_{2,5} & N_{2,6} \\
N_{3,1} & N_{3,2} & N_{3,3} & N_{3,4} & N_{3,5} & N_{3,6} \\
N_{4,1} & N_{4,2} & N_{4,3} & N_{4,4} & N_{4,5} & N_{4,6}
\end{array}\right]\left[\begin{array}{c}
q_{1} \\
q_{2} \\
q_{3} \\
q_{4} \\
q_{5} \\
q_{6}
\end{array}\right] .
$$

Once the shape functions were obtained, the discretized forms of $u_{1}, u_{2}, w_{1}$, and $w_{2}$ (Eq. 9) were inserted into the energy equation (Eq. 1). The $i, j^{\text {th }}$ component of the stiffness, $k_{i, j}$, was found through the following derivative:

$$
k_{i, j}=\frac{\partial^{2}}{\partial q_{i} \partial q_{j}} U
$$

The stiffness matrix was then included in a custom finite element solving routine and combined with standard beam elements (Fig. 1). The stresses in the adhesive were then found using Eqs. 7.

Since the purpose of creating a macroscopic joint element is to provide a means of integrating a fast closed-form solution into vehicle-scale structural finite element models, simplifying assumptions are very desirable to make the formulation easier and faster. Additionally, since cohesive zone type failure models which require iterative solving 
will be added to the joint element in the future, it is even more vital that the equations are as simple as possible. However, simplifying assumptions are often limiting, and it is important to know these limitations. Therefore, three models created by applying different simplifying assumptions about the stress state in the adhesive layer were compared in order to determine which set of assumptions should be used in the joint element, for different joint parameters.

\section{A. Model 1}

Model 1 is the baseline model with no further simplifying assumptions other than those already stated in the analytical formulation. The adhesive is assumed to be in a state of plane stress, and the stress and strain are defined in Eqs. 6 and 7.

\section{B. Model 2}

In this model, a widely-used assumption ${ }^{4,6,7,8}$ which neglects the adhesive stress $\sigma_{a x x}$ compared to the peel stress $\sigma_{a z z}$ and the shear stress $\tau_{a x z}$, is adopted. The strain in the adhesive is defined as before:

$$
\begin{gathered}
\gamma_{a x z}=\left(\frac{\partial}{\partial z_{a}} u_{a}\left(x, z_{a}\right)\right)+\left(\frac{\partial}{\partial x} w_{a}\left(x, z_{a}\right)\right) \\
\varepsilon_{a z z}=\frac{\partial}{\partial z_{a}} w_{a}\left(x, z_{a}\right)
\end{gathered}
$$

while the stress is defined simply as:

$$
\begin{gathered}
\tau_{a x z}=G_{a} \gamma_{a x z} \text { and } \\
\sigma_{a z z}=E_{a} \varepsilon_{a z z}
\end{gathered}
$$

This model is very similar to modeling the adhesive as a bed of linear shear and normal springs, except that the second term in the shear strain equation (Eq. 6a) couples the normal springs to the shear springs.

\section{Model 3}

In this model, it is assumed that the adhesive is a bed of uncoupled linear shear and normal springs. The adhesive layer is assumed to be so thin that the stress in the adhesive layer is independent of the $z$-coordinate. The strain for this model is defined as:

$$
\begin{gathered}
\gamma_{a x z}=\frac{\partial}{\partial z_{a}} u_{a}\left(x, z_{a}\right) \text { and } \\
\varepsilon_{a z z}=\frac{\partial}{\partial z_{a}} w_{a}\left(x, z_{a}\right)
\end{gathered}
$$

while the stress is defined as in Model 2 (Eqs. 7a and 11).

\section{Model Implementation}

Two studies were done to validate the macroscopic joint element and discover the simplest model required to accurately predict the shear and peel stress in a single lap joint. First, the models were compared with each other over a broad range of joint geometric parameters to show which models have an impact on the predicted stress for different parameter values. Second, a 2-D solution, based on the finite element method (FEM) was generated for four parameter cases and the predicted adhesive stresses were compared with the three models. This was done to illustrate the accuracy of the macroscopic joint element for different geometric parameters.

The three models were compared over a range of parameters to determine the difference in peak shear and peel stress along the adhesive centerline $\left(z_{a}=\eta / 2\right)$ predicted by the models as a function of the parameters. This is useful to show when assumptions about the adhesive stresses are valid and when one should be careful when using them. Figure 5 shows the dimensions and material properties of the single lap joint considered. The adherends were 
aluminum, with a modulus of $70 \mathrm{GPa}\left(E_{1}\right.$ and $\left.E_{2}\right)$ and Poisson's ratio of $0.33\left(v_{1}\right.$ and $\left.v_{2}\right)$. They were both $5 \mathrm{~mm}$ thick $\left(t_{l}\right.$ and $\left.t_{2}\right), 2 \mathrm{~mm}$ wide $(b)$, and extended $5000 \mathrm{~mm}$ long past the joint overlap. The adhesive was FM300 and had a modulus of $2.17 \mathrm{GPa}\left(E_{a}\right)$ with a shear modulus of $0.89 \mathrm{GPa}\left(G_{a}\right)$. The adhesive thickness $(\eta)$ was varied from 0.005 to $5 \mathrm{~mm}$, and the overlap length $(l)$ was varied from 50 to $5000 \mathrm{~mm}$. Although these parameters are not necessarily typical for a joint, it was necessary to test a wide range of parameters to demonstrate the working range of the macroscopic element and the limits of the assumptions.

On either side of the macroscopic joint element, 50 beam elements were used to model the adherends outside of the overlap region. The left end was clamped, or restrained from displacement. The right end was extended in the $x$ direction by $10 \mathrm{~mm}$ and restrained from rotating and from displacing in the $z$-direction as shown in Figure 5 .

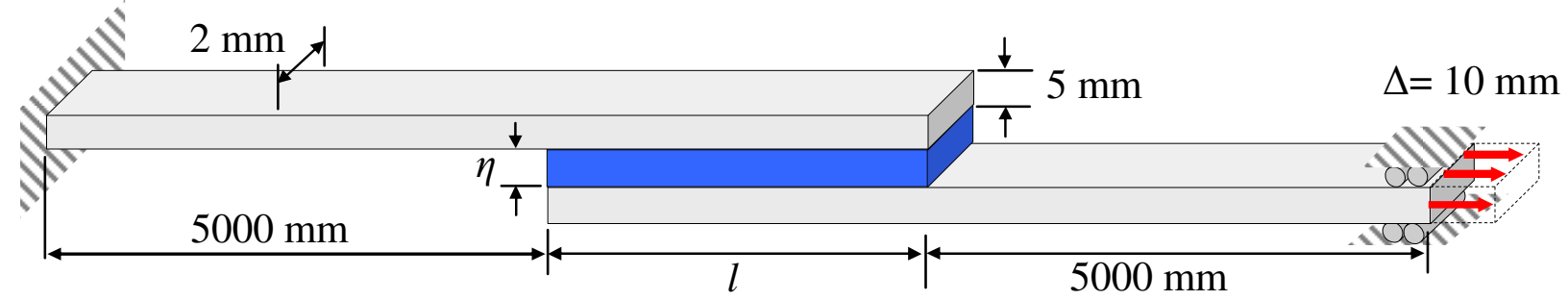

Figure 5. Boundary conditions, loading scenario and geometric parameters for the single lap joint. Material properties include: $E_{1}=E_{2}=70 \mathrm{GPa}, E_{a}=2.17 \mathrm{GPa}$, and $\mathrm{G}_{\mathrm{a}}=0.89 \mathrm{GPa}$.

Along with comparing the three models to each other, they were also compared with the results from a 2-D FEM created using the commercial package Abaqus ${ }^{10, \sharp}$ for four different geometric parameter cases. The same geometric parameters and boundary conditions shown in Fig. 5 were used for the cases, and Table 1 shows the values of 1 and $\eta$ used for each case. Cases were chosen at four extreme corners of the parameters tested for the comparison study.

Table 1. Parameters of in-depth study cases.

\begin{tabular}{lcc}
\hline & $\begin{array}{c}\text { adhesive } \\
\text { length }(\boldsymbol{l}) \mathbf{\text { mm }}\end{array}$ & $\begin{array}{c}\text { adhesive } \\
\text { thick. }(\boldsymbol{\eta}), \mathbf{m m}\end{array}$ \\
\hline Case 1 & 5000 & 5 \\
Case 2 & 5000 & 0.5 \\
Case 3 & 50 & 5 \\
Case 4 & 50 & 0.5 \\
\hline
\end{tabular}

The finite element models were constructed using 70,000 to 100,000 2-D plane strain quadrilateral elements, with an element bias towards the corners of the adhesive. One issue which deserves mention is that the macroscopic joint element models do not fulfill the traction free $\left(\sigma_{a x x}\right.$ and $\left.\tau_{a x z}\right)$ boundary condition at the free edges of the adhesive boundary. Therefore, the FEMs and the macroscopic joint element models are not expected to predict similar stresses at the free edges of the adhesive. Moreover, the inside corners of the adhesive in the FEM causes a stress singularity, making the model mesh dependant in the corner singularity region. In application, spew fillets are applied to eliminate this stress concentration and cause the edges of the overlap to not have zero stress ${ }^{7}$. Therefore it is of no major concern that the macro joint element does not reflect the stresses predicted by the FEA model at the ends of the adhesive. Since the model is meant to serve as an initial vehicle-scale model element for initial sizing and not necessarily for detailed analysis, the goal of the validation is to show that the overall behavior of the joint is reflected by the macroscopic joint element.

\footnotetext{
\# Abaqus is a commercial finite element package under license to the University of Michigan.
} 


\section{Results and Discussion}

The plots comparing the relative difference between the maximum centerline peel and shear stresses predicted by Models 1 and 2 for different $\eta / t$ and $t / l$ values can be found in Fig. 6 . Model 2 was created by taking model 1 , and assuming that the extensional stress, $\sigma_{a x x}$, was negligible. The relative difference between the maximum peel stress predicted by Models 1 and 2 is between $8 \%$ and $5 \%$ for the whole range of adhesive thicknesses and overlap lengths considered. The difference is greatest for the very thin adhesive layers, and least for the thickest adhesive layers. Additionally, the difference between the maximum adhesive centerline shear stress predicted by the two models is below $1 \%$ for all of the parameters considered in this study. Considering the uncertainty of the stress state at the corner of the adhesive due to the previously mentioned issues related to stress concentrations and violation of the traction free condition, the difference between these models is very low. If one is only concerned with the peel and shear stress in the adhesive layer, it would be advantageous to use Model 2 over Model 1 due to its increased simplicity. However, Tsai and Morton ${ }^{12}$ note that the magnitude of $\sigma_{a x x}$ near the end of the overlap is often comparable to the magnitude of the peel and shear stresses. Therefore, it should be kept in mind that Model 1 is the only one of the three models to yield the extensional stress, $\sigma_{a x x}$.

Model 3 was created by taking Model 2 and uncoupling the transverse and axial displacements. In other words, Model 3 assumed that the stress and strain in the adhesive layer is constant in the z-direction. Fig. 7 contains a comparison of the relative difference between the maximum centerline peel and shear stresses predicted by Models 2 and 3 for different $\eta / t$ and $t / l$ values. It can be seen that these models predict very different values of maximum stress. The shear stress difference can reach up to $40 \%$, while the peel stress difference can be almost $30 \%$. It appears that the differences between the two models are especially large for thicker adhesive layers and shorter overlaps. Interestingly, the sign change between $t / l$ values of .001 and .01 in both plots indicates that there may be a $t / l$ value which causes the difference to hover around zero.
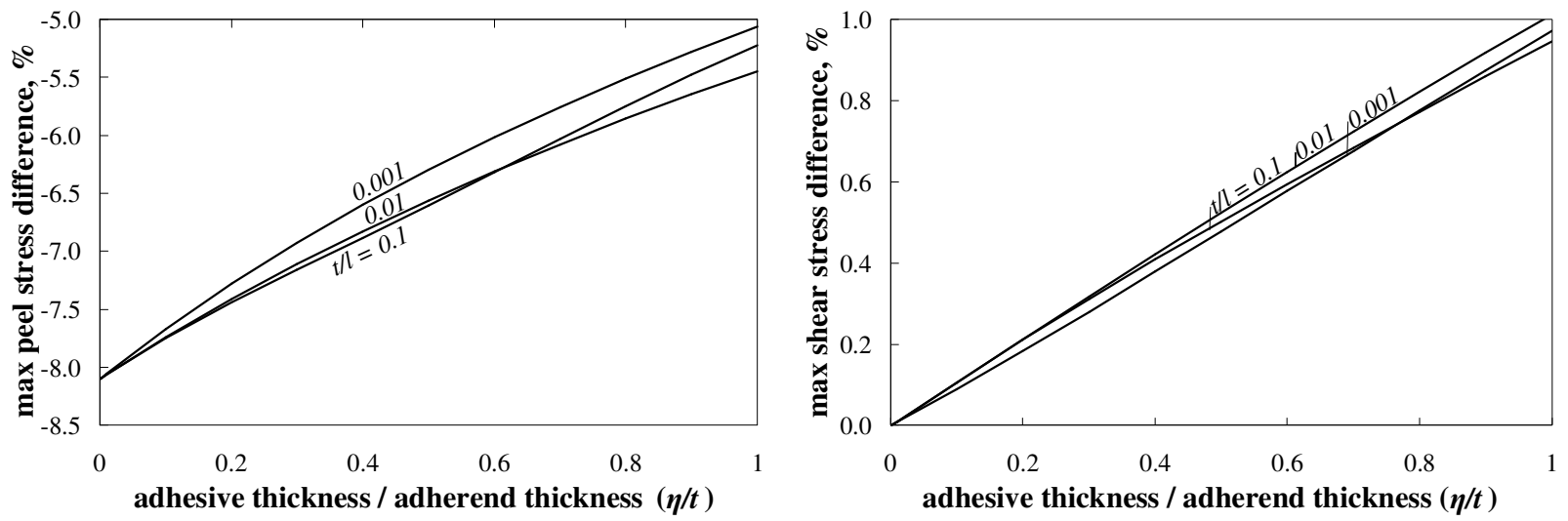

Figure 6. Relative difference in maximum adhesive centerline $\left(z_{a}=\eta / 2\right)$ shear and peel stress between Models 1 and 2.
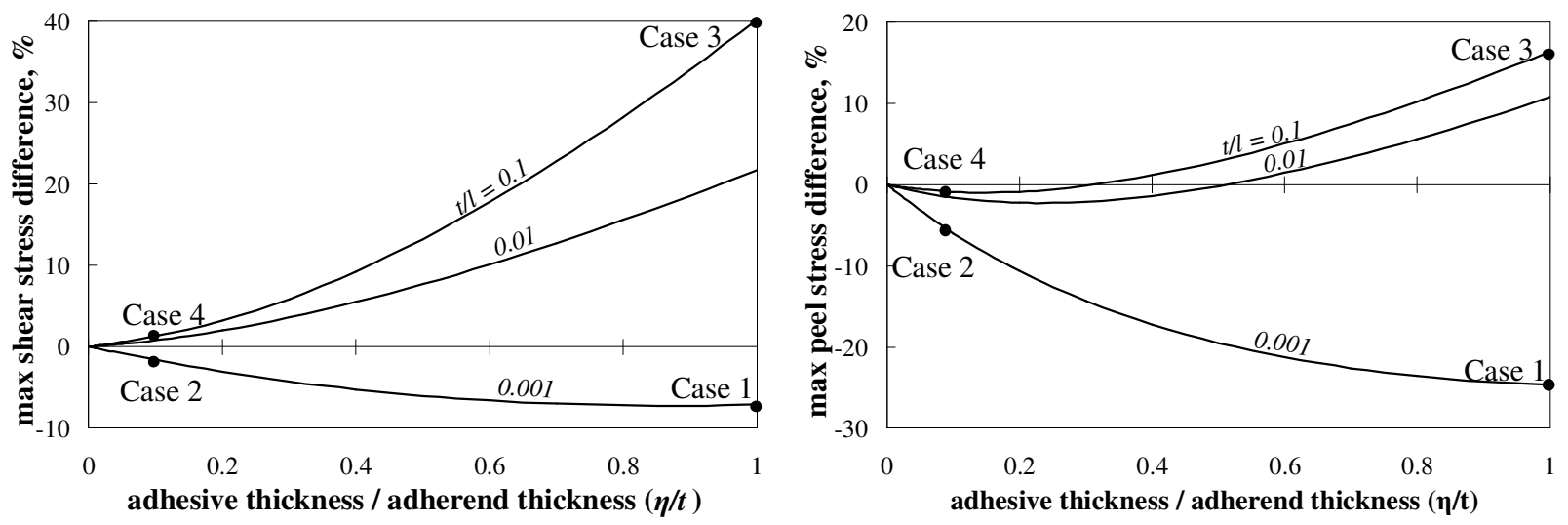

Figure 7. Comparison of the maximum shear and peel stress predicted by Models 2 and 3 for different joint configurations, along with points indicating the cases for comparison with 2-D FEMs. 
2-D plane stress FEMs were constructed for four cases, each case with parameters as described in Table 1 and the points labeled in Fig. 7. The results of the comparison between the FEM models and the macro joint element models are found in Figs. 8-15. Figs. 8, 10, 12, and 14 compare the adhesive centerline stresses of the three models with the FEM for Cases 1-4. 2-D contour plots showing the shear and normal stress distribution in the adhesive as predicted by the FEM and three models for each case are shown in Figs. 9, 11, 13, and 15.

\section{A. Case 1}

Case 1 had a thick, long adhesive layer with $\eta / t=1$ and $t / l=0.001$. The length of the adhesive displayed in Figs. 8 and 9 is only $0.5 \%$ of the length because the adhesive in the middle region is effectively stress free, which makes it this region unimportant for the current study. Obviously, this joint would be very inefficient because almost all of the stress is held by less than $1 \%$ of the adhesive. All three models predict the peel stress fairly well, although Models 1 and 2 appeared to be slightly more accurate. Looking at the adhesive peel stress distribution in Fig. 9, Models 2 and 3 predict constant peel stress in the $z$-direction, while the peel stress distribution of the 2-D FEM varies nonlinearly in the z-direction, even past the free end.
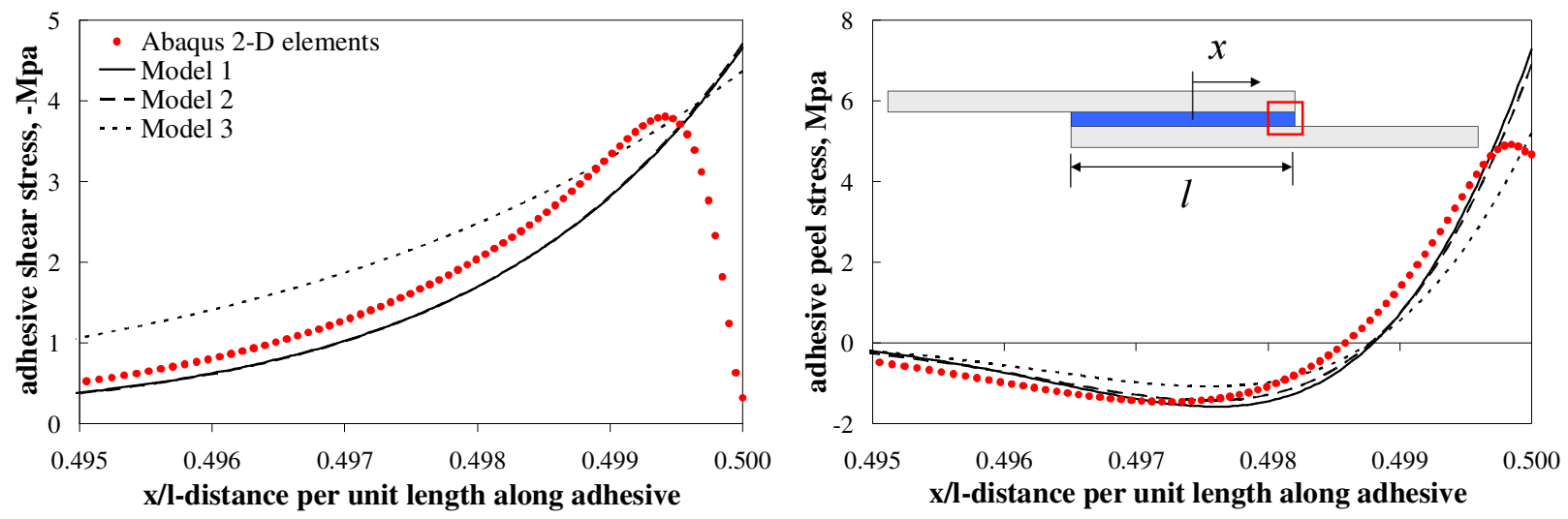

Figure 8. Adhesive centerline stresses predicted by different models for Case 1 parameters.
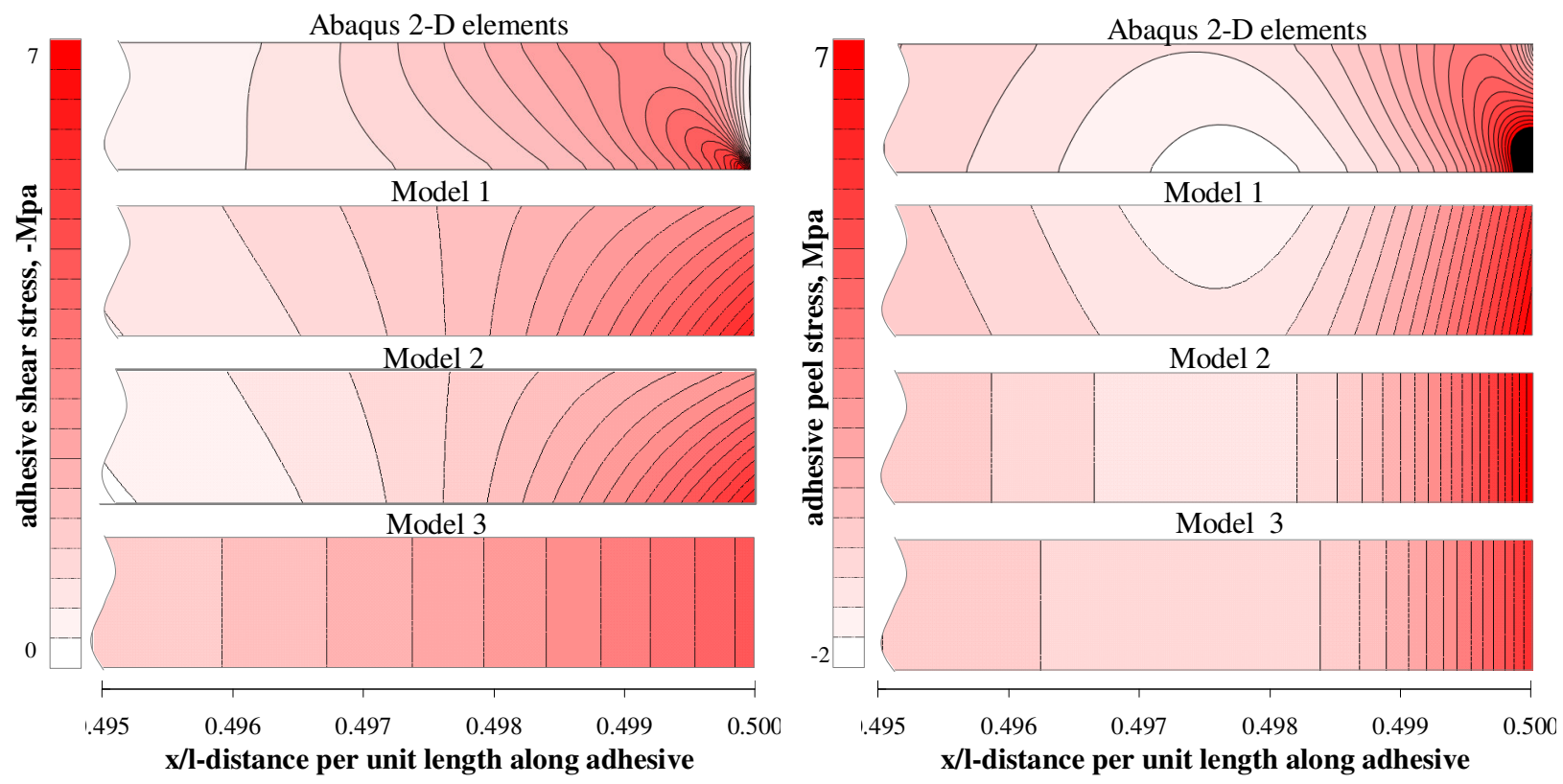

Figure 9. Adhesive stress contours predicted by different models for Case 1 parameters. 
Looking at the centerline shear stress, Model 2 does not seem to match up with the FEM very well. While Models 1 and 2 are low, they appear to capture the general trend. Obviously, none of the models reflect the free end condition of zero shear traction. The shear stress contour plots in Fig. 10 show that Model 1 has shear stress constant in the $z$-direction, but the FEM model shows that the stress varies significantly in the $z$-direction, which might explain the inaccuracy of the model. The distribution of shear stress in Models 1 and 2 is a closer match to the FEM model, although the slopes appear to be incorrect past the free end.

\section{B. Case 2}

Case 2 was composed of a joint with a thin, long adhesive layer $(\eta / t=0.1$ and $t / l=0.001)$. Like Figs. 9 and 10 , the length of the adhesive displayed in Figs. 11 and 12 is only $0.5 \%$ of the length because only the ends display behavior worth comparison. All three models predict the peel and shear stress very well. The reason why can be seen in the contour plots in Fig. 12. The non-linear distribution of stress at the free end of the adhesive predicted by the 2-D FEM dies out very quickly, and the remainder of the adhesive has a linear distribution in the $z$-direction. Since the thickness of the adhesive is so small, the stress can be effectively modeled as constant in the $z$-direction. Therefore, using the simplified Model 3 would still yield very accurate results for this case.
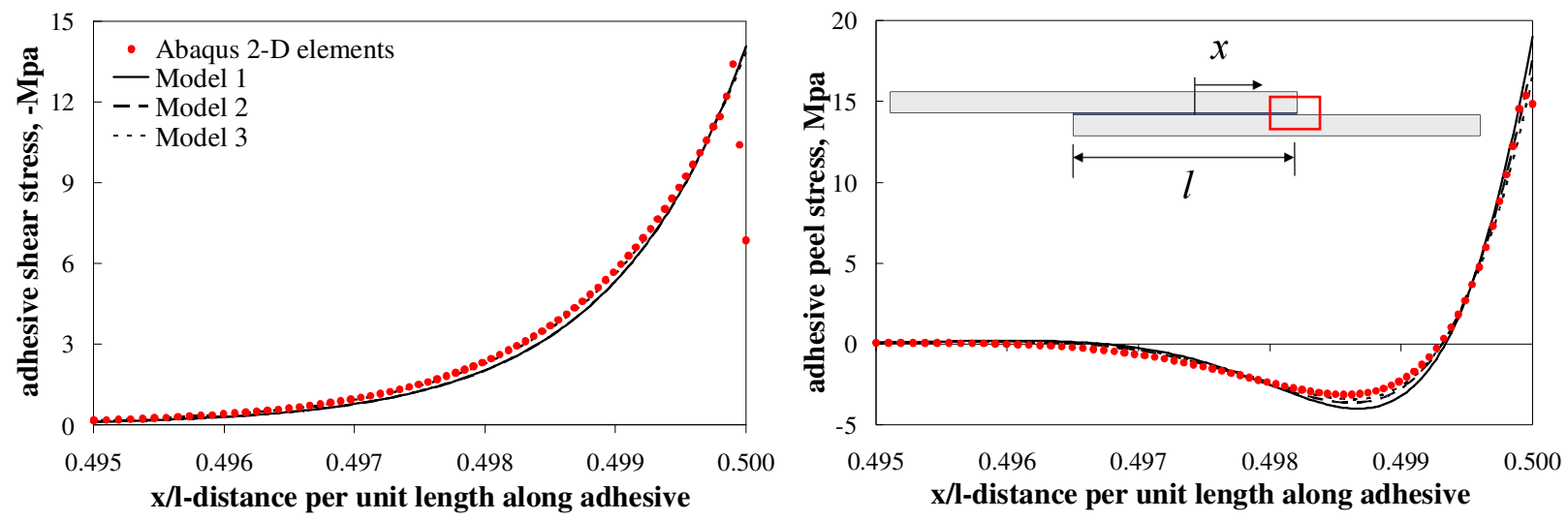

Figure 11. Adhesive stress contours predicted by different models for Case 2 parameters.
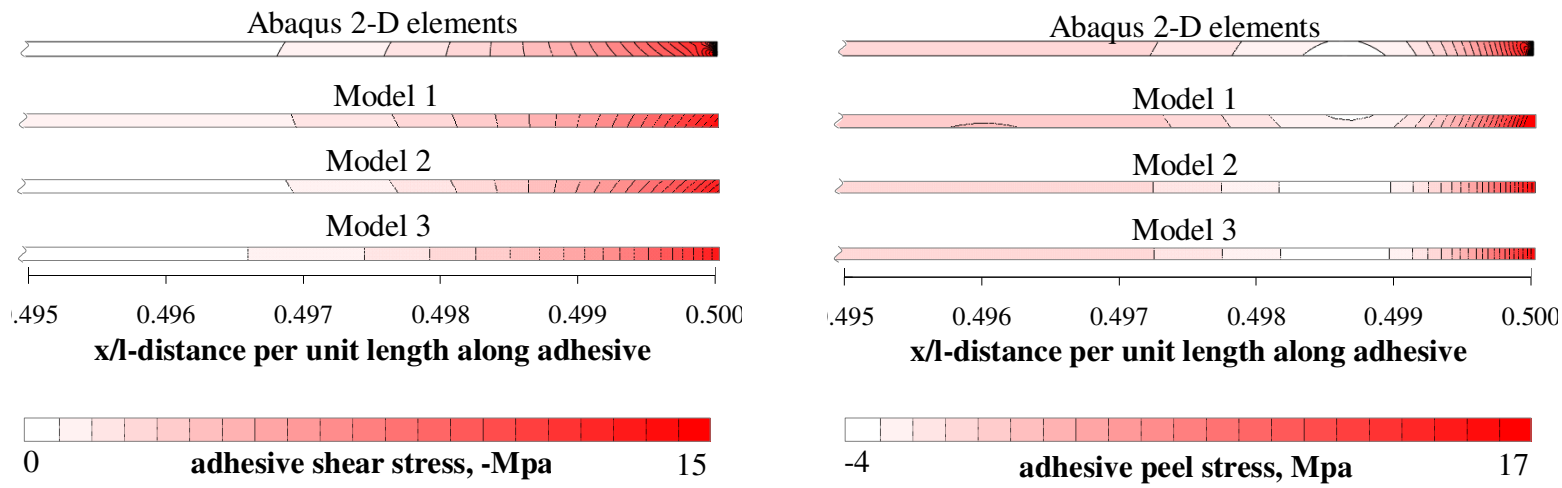

Figure 10. Adhesive centerline stresses predicted by different models for Case 2 parameters.

\section{Case 3}

Case 3 had a thick, short adhesive layer with $\eta / t=1$ and $t / l=0.1$. The right half of the adhesive is shown in the plots. The three model predictions of the shear stress were not very consistent with the 2-D FEM. Model 3 vastly over predicts the stress levels, and Models 1 and 2 under predict the shear stress. Looking at the shear stress distribution of the FEM in Fig. 12, the stress is non-linear in the $z$-direction and the effects of the free end do not die out quickly as with the thin adhesive cases. The constant $z$-direction stress distribution of Model 3 is insufficient to correctly model the adhesive, especially when it comes to the shear stress.

The peel stress, on the other hand, was not so poorly predicted. All three models predicted similar trends for the adhesive centerline peel stress, even though the prediction appears to lag behind the stress predicted by the 2-D 
FEM. Generally, Model 3 would be a poor choice for a joint of this type. Models 2 and 3 are closer, but still do not accurately predict the stress levels of the single lap joint for Case 3.
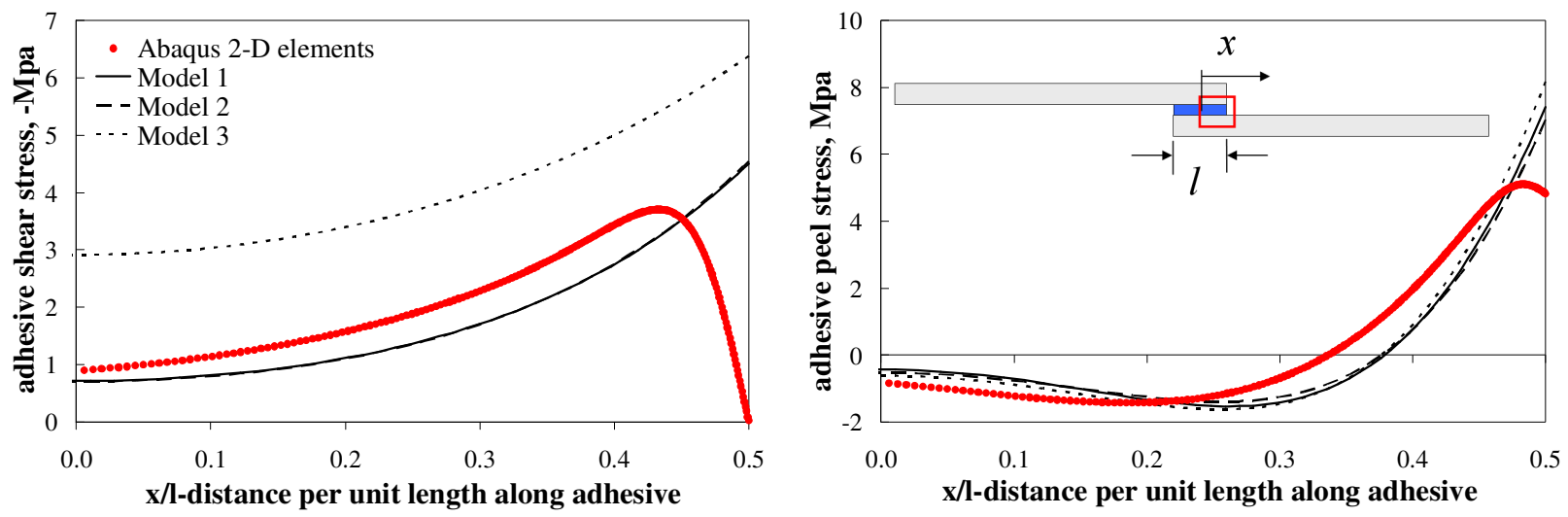

Figure 12. Adhesive centerline stresses predicted by different models for Case 3 parameters.
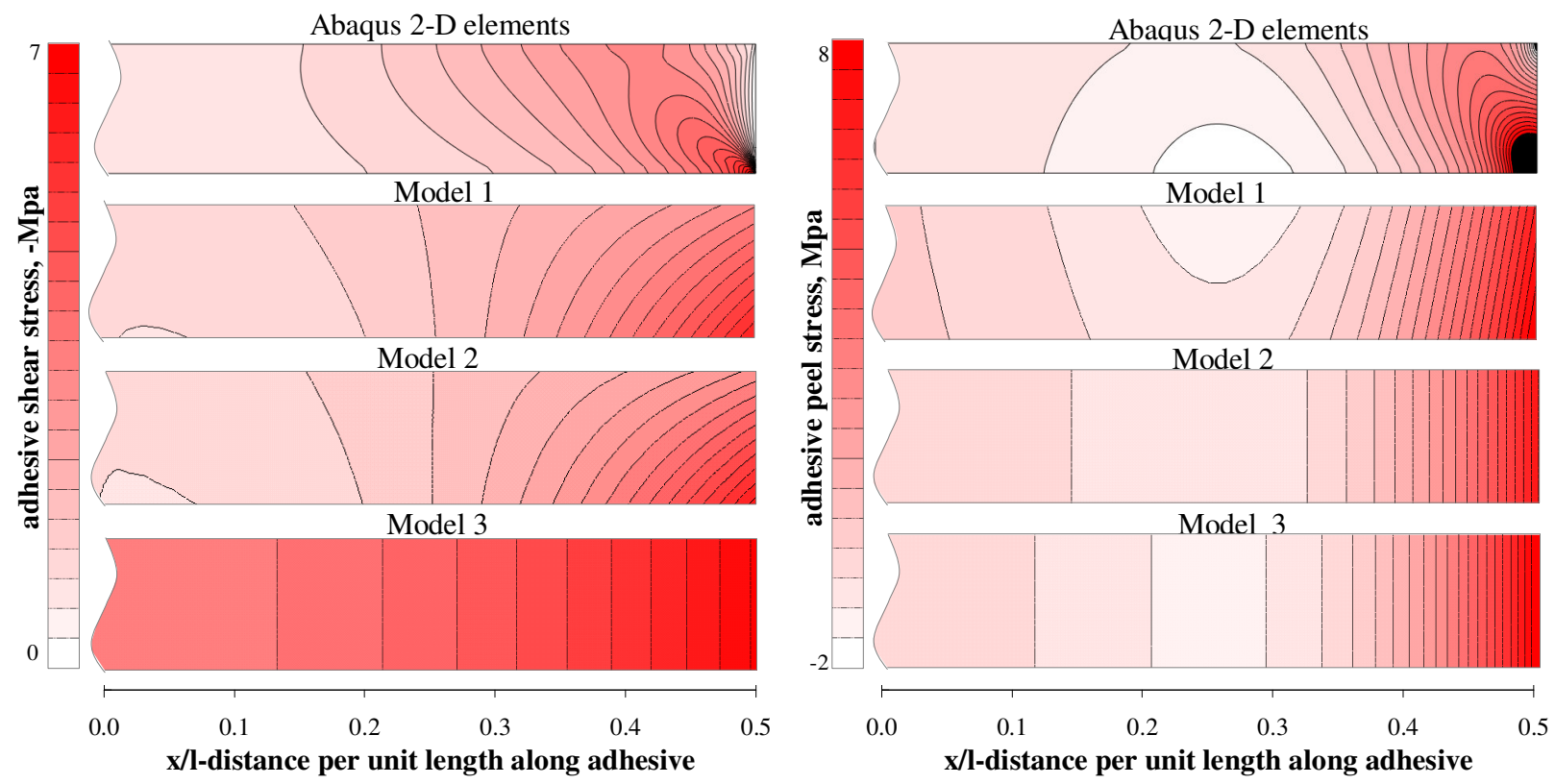

Figure 13. Adhesive stress contours predicted by different models for Case 3 parameters.

\section{Case 4}

Case 4 was composed of a joint with a thin, short adhesive layer $(\eta / t=0.1$ and $t / l=0.1)$. All three models under predicted the shear stress slightly, and surprisingly, Model 3 is the closest to the 2-D FEM. The contour plot of the FEM shear stress in Fig. 15 shows that the effects of the free edge disappear quickly in the adhesive. The shear stress distribution varies linearly in the $z$-direction, but Models 1 and 2 have the wrong slopes, which probably accounts for the inaccuracy. Since Model 3 has no slope, it is slightly more accurate than Models 1 and 2. The models predicted the FEM peel stress slightly more accurately, but there was still a lag similar to Cases 1 and 3 . It is unclear what causes the lag; whether it is an effect created by the difference in free edge conditions, or simply due to inaccuracies in the model.

It should be kept in mind that the macroscopic joint element is not meant for detailed stress analysis of the joint, unless more refinement and inclusion of non-linear constitutive properties of the adhesive leads to joint failure predictions, a task that is currently underway. The models presented here reflect the behavior of the joint in a global sense to be used for early sizing studies. When one keeps that in mind, the stress states predicted by the models for 
all of the cases appear to be fairly accurate. The models are especially suited for thin adhesive layers, and appear to be even more accurate for long, thin adhesive layers.
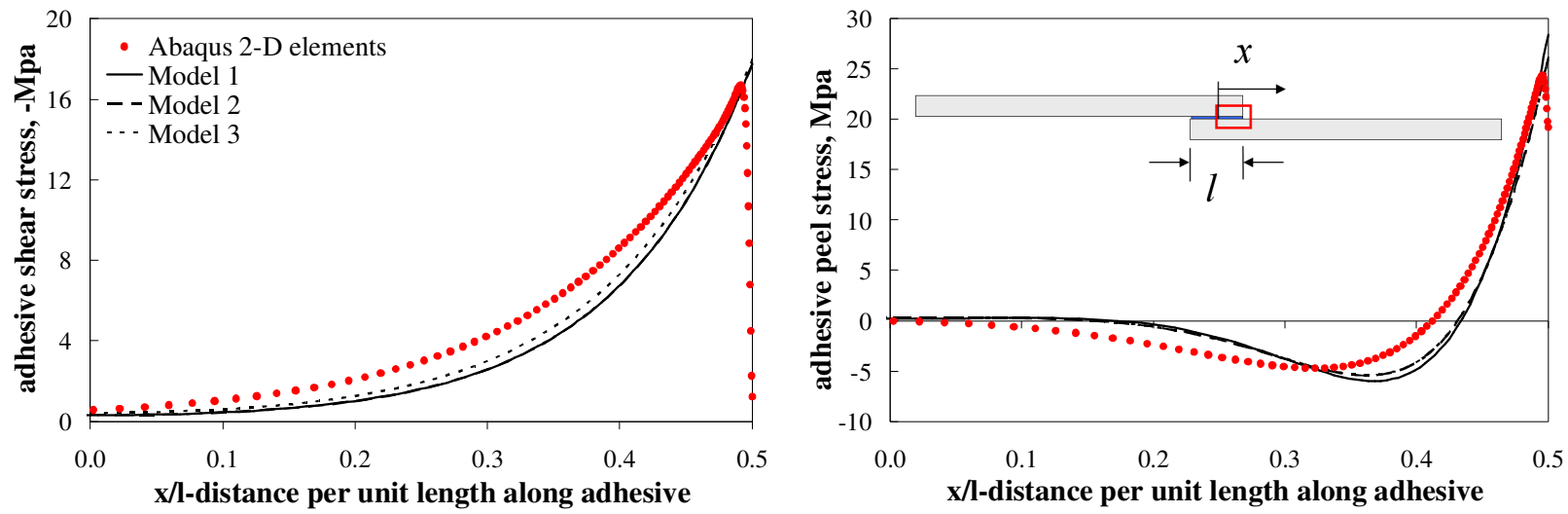

Figure 14. Adhesive centerline stresses predicted by different models for Case 4 parameters.
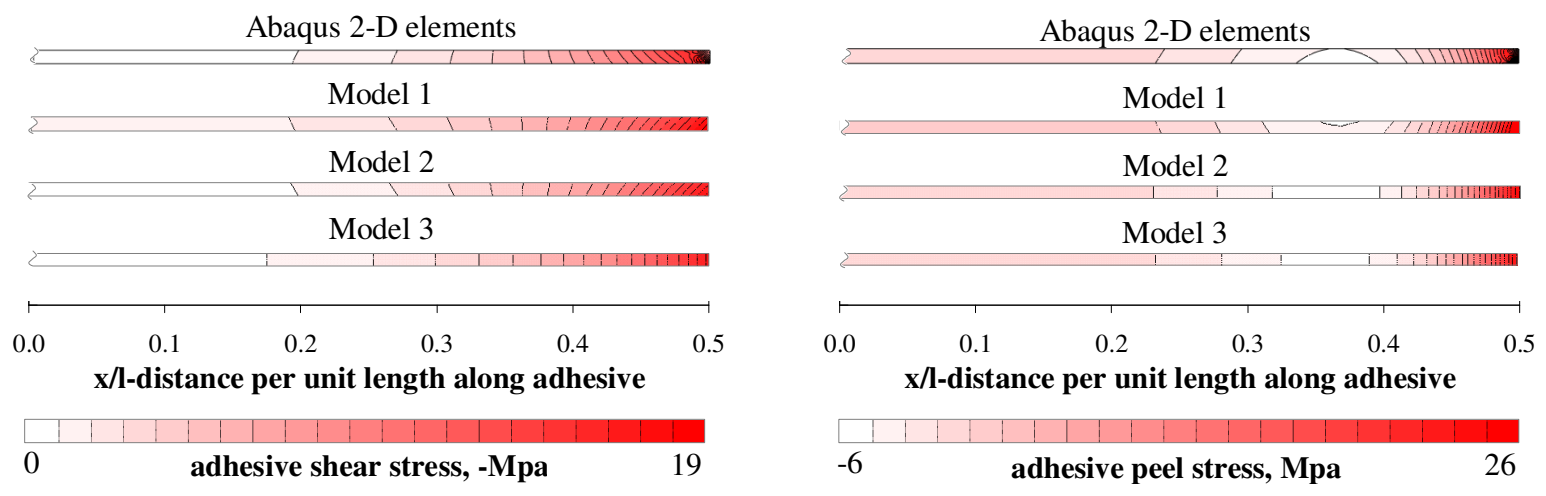

Figure 15. Adhesive stress contours predicted by different models for Case 4 parameters.

\section{Summary and Concluding Remarks}

Eccentric load paths caused by single lap joints often require a detailed, dense mesh in structural finite element models of assemblies containing single-lap joints, which can be costly for global, vehicle scale models. To reduce computational time required for these large-scale models and aid joint sizing early on in the design phase, a macroscopic element was created to model the behavior of a joint with a single element. This was accomplished by embedding an approximate analytical solution of the single lap joint into a finite element. The current model assumes that all materials remain linearly elastic, but the next step is to add nonlinear constitutive failure models to predict progressive adhesive failure. Since the next generation progressive failure element will have to be solved iteratively, it is imperative that the simplest formulation be used to reduce the complexity and computing time required for each iteration. With this in mind, three versions of the macroscopic joint element were created, each containing additional simplifying assumptions about the stress state of the adhesive.

The first version, Model 1, assumes that the adhesive is in plane stress, and considers $\sigma_{a x x}, \sigma_{a z z}$, and $\tau_{a x z}$. Model 2 ignores $\sigma_{a x x}$. Finally, Model 3 ignores $\sigma_{a x x}$ like Model 2, but it also assumes that the stresses within the adhesive are independent $z$-direction. The maximum peel and shear stress predicted by these three models was compared to ascertain how much of a difference each additional assumption matters. It was found that there was little difference between Models 1 and 2. Since Model 2 is simpler than Model 1, it can be concluded that Model 1 is unnecessary, unless the value of $\sigma_{a x x}$ is desired. On the other hand, Models 2 and 3 predicted very different maximum stresses, especially for joints with thick adhesive layers and short joint overlap lengths.

Four joints with different combinations of thick and thin adhesive layers and long and short overlap lengths were studied in depth and compared with 2-D finite element models to 1) identify which models were more accurate, and 
under what parameter conditions and 2) to show that the macroscopic joint finite element could be used to give a general prediction of joint behavior. It was found that all of the models were more accurate for joints with thin adhesive layers. Also, all of the models were slightly more accurate for longer joint overlaps. Therefore, for thin joints, Model 3 should be used because it can get an answer just as accurate as the other models, with a much simpler formulation. For thick joints, Model 2 would be preferred because it is more accurate than Model 3, although the models are generally not as accurate for thick joints. However, most joints in application have very thin adhesive layers, so Model 3 should be adequate for most real-life situations. It was also shown that for all cases, the macroscopic joint elements were more than adequate at predicting the behavior of a joint for early design sizing purposes.

\section{Acknowledgements}

This work was financially supported by the Space Vehicle Technology Institute under grant NCC3-989 jointly funded by NASA and the Department of Defense. It is managed within the NASA Constellation University Institutes Project, with Claudia Meyer as the project manager and H. Kevin Rivers \& Stanley Smeltzer (NASA Langley), as the project monitors. The interest and encouragement of Steve Arnold and Brett Bednarcyk, NASA Glenn, are gratefully acknowledged.

\section{References}

${ }^{1}$ Hart-Smith, L., “Adhesive Bonding of Composite Structures- Progress to Date and Some Remaining Challenges,” Journal of Composites Technology and Research, Vol. 24, No. 3, 2002, pp. 133-151.

${ }^{2}$ Frostig, Y., Thomsen, O., Mortensen, F., "Analysis of Adhesive-Bonded Joints, Square-End, and Spew-Fillet- High-Order Theory Approach,” Journal of Engineering Mechanics, Vol. 125, No. 11, 1999, pp. 1298-1307.

${ }^{3}$ Gustafson, P. A. and Waas, T. A. "A Macroscopic Finite Element for a Symmetric Doublelap Joint Subjected to Mechanical and Thermal Loading," Proceedings of the AIAA/ASME/ASCE/AHS/ASC $48^{\text {th }}$ Structures, Structural Dynamics and Materials Conference, April 23-26, 2007, Honolulu, HI, No. 2007-2308, American Institute of Aeronautics and Astronautics, 2007.

${ }^{4}$ Volkersen, O., "Die Nietkraftverteilung in Zugbeanspruchten mit Konstanten Laschenquerschritten," Luftfahrtforschung, Vol 15, 1938, pp. 41-47.

${ }^{5}$ Goland, M. and Reissner, E. "The Stresses in Cemented joints," Journal of Applied Mechanics, Vol. 11, 1944, pp A17-A27.

${ }^{6}$ Hart-Smith, L. J. “Adhesive bonded single lap joints," Tch. Rep. NASA CR 112236, Douglas Aircraft, McDonnel Douglas Corporation, Long Beach, California, 1973.

${ }^{7}$ Mortensen, F. and Thomsen, O.T., "Analysis of Adhesive Bonded Joints: a Unified Approach,” Composites Science and Technology, Vol. 62, 2002, pp. 1011-1031.

${ }^{8}$ Delale, F., Erdogan, F., and Aydinoglu, M. N., "Stresses in Adhesively Bonded Joints: A Closed-Form Solution," Journal of Composite Materials, Vol. 15, 1981, pp. 249-271.

${ }^{9}$ Zhang, J, Bedmarcyk, B. A., Collier, C, Yarrington, P., Bansal, Y., and Pindera, M. J., "3D Stress Analysis of Adhesively Bonded Composite Joints," Proceedings of the AIAA/ASME/ASCE/AHS/ASC $46^{\text {th }}$ Structures, Structural Dynamics and Materials Conference, April 18-21 2005, Austin, Texas, No. 2005-2021, American Institute of Aeronautics and Astronautics, 2005.

${ }^{10}$ ABAQUS, Inc, ABAQUS user Manuel v6.7-1, Electronic Version, 2006.

${ }^{11}$ Tsai, M. Y. and Morton, J., "An evaluation of Analytical and Numerical Solutions to the Single-Lap Joint," Int. J. Solids Structures, Vol. 31, No. 18, 1994, pp. 2537-2563. 